\title{
Self-organized, noise-free escape of a coupled nonlinear oscillator chain
}

\author{
D. Hennig ${ }^{1}$, L. Schimansky-GeieR ${ }^{1}$ and P. HäNGGI ${ }^{2}$ \\ ${ }^{1}$ Institute of Physics, Humboldt-University at Berlin - Newton Str. 15, D-12489 Berlin \\ ${ }^{2}$ Institut für Physik, Universität Augsburg - Universitätsstr. 1, D-86135 Augsburg, Germany
}

received 24 January 2007; accepted in final form 1 March 2007

published online 28 March 2007

\author{
PACS 05.40.-a-Fluctuation phenomena, random processes, noise, and Brownian motion \\ PACS 05.65.+b - Self-organized systems \\ PACS 87.15.-v - Biomolecules: structure and physical properties
}

\begin{abstract}
We consider the self-organized escape of a chain of harmonically coupled units from a metastable state over a cubic potential barrier. The underlying dynamics is conservative and purely deterministic. The supply of a sufficient total energy transforms the chain into the nonlinear regime from which an initially, nearly uniform lattice configuration becomes unstable, yielding a redistribution with a strong localization of energy. A spontaneously emerging localized mode grows into the critical nucleus. Upon passing this transition state, the nonlinear chain performs a collective, deterministic escape event. Surprisingly, we find that such noise-free, collective nonlinear barrier crossing events yield a drastically diminished average escape time as compared to the one that is assisted by continuously impacting thermal noise. This beneficial enhancement of the rate of escape for the chain typically occurs whenever the ratio between the average energy supplied per unit in the chain and the potential barrier energy assumes small values.
\end{abstract}

Copyright (C) EPLA, 2007

Ever since the benchmark work by Kramers (for a comprehensive review see ref. [1]), there is continued and growing interest in the dynamics of escape processes of single particles, of coupled degrees of freedom or of small chains of coupled objects out of metastable states. It is realized by the passage of the considered objects over an energetic barrier which separates the local potential minimum from a neighboring attracting domain.

A common situation in statistical physics is that of a stochastic escape for which the total energy remains a constant on average only. The later circumstance assumes the existence of a thermal bath, causing dissipation and local energy fluctuations. Thus, in this situation the escape necessitates the creation of an optimal fluctuation triggering the escape [1]. Put differently, when such an optimal fluctuation is transferred to the chain it provides sufficient energy to the chain to statistically overcome the energetic bottleneck. Characteristic time-scales of these processes are determined by the calculation of corresponding rates of escape out of the corresponding domain of attraction. Consequently, many generalizations of Kramers escape theory [1] in over- and underdamped versions have been widely exploited. First extensions to multi-dimensional systems date back to the late '60s [2]. Nowadays, this method is commonly utilized in biophysical contexts and for a great many applications occurring in physics and chemistry [3-10].

The objective of this letter is to elaborate on a different scenario of the possible exit from a metastable domain of attraction; the main mechanism is based on the assistance of a strongly nonlinear deterministic dynamics. The model we shall investigate is a purely deterministic dynamics of a bi-linearly coupled chain of nonlinear oscillators. Thus, no additional coupling to a thermal bath assists the escape. This set-up thus implies a vanishing dissipation. We consider macroscopic discrete, coupled nonlinear oscillator chains with up to 1000 links, as these may appear as realistic models in neuroscience, in various biophysical contexts or also in networks of coupled superconductors, e.g. see refs. [11-14]. An efficient deterministic escape that is driven in the absence of noise is particularly important when dealing with low temperatures for which the activated escape becomes far too slow, or also for situations with many coupled nonlinear units in the presence of non-thermal intrinsic noise that scales inversely with the square root of the system size. If the chain is brought into the nonlinear regime it may exhibit a spontaneous energy localization. This is due to a modulational instability [15] 
of the homogeneous chain in the metastable state and the possibly subsequent interaction of breather solutions.

An escape is related with a crossing of a saddle point in configuration space, corresponding to bottlenecks [1]. We shall assign a potential energy of interaction and external force for this transition state $E_{c r i t}$ which has has to be concentrated at the critical mode. We intend to show that the latter can be reached in the microcanonical situation spontaneously. Hence we encounter a selforganized creation of critical states in clear contrast to noise-activated escape. In particular, we find that intrinsic nonlinear effects on a long discrete chain of $N$ units induces a transition over an energetic barrier by enhancing one, or several localized breather states $[13,16,17]$. With this mechanism the initially almost uniformly distributed energy can become dynamically concentrated by internal redistribution without the need of an assistance of energy exchange with a thermal bath. With this work we demonstrate that the mechanism of nonlinear energy localization may promote a faster escape dynamics as compared to the noise-assisted situation where the system experiences a continual stochastic forcing.

Each chain member is positioned in a cubic, metastable potential,

$$
U(q)=\frac{\omega_{0}^{2}}{2} q^{2}-\frac{a}{3} q^{3}
$$

where $a>0$ measures the nonlinearity. The metastable equilibrium of the potential is located at $q_{\min }=0$ and the maximum is at $q_{\max }=\omega_{0}^{2} / a$. Each nonlinear oscillator is harmonically coupled to its neighbors. The situation is thus modeled by a one-dimensional lattice system of $N$ coupled oscillators with the total Hamiltonian reading:

$$
H=\sum_{n=1}^{N}\left\{\frac{p_{n}^{2}}{2}+U\left(q_{n}\right)\right\}+\frac{\kappa}{2} \sum_{n=1}^{N}\left[q_{n+1}-q_{n}\right]^{2} .
$$

The coordinate $q_{n}$ quantifies the position of the $n$-th unit from a rest value in the direction perpendicular to the axis of the chain and $p_{n}$ denotes the canonically conjugated momentum. Throughout this work we shall impose periodic boundary conditions.

The deterministic dynamics of the coupled oscillators emerges as

$$
\frac{\mathrm{d}^{2} q_{n}}{\mathrm{~d} t^{2}}+\omega_{0}^{2} q_{n}-a q_{n}^{2}-\kappa\left[q_{n+1}+q_{n-1}-2 q_{n}\right]=0
$$

The chain is linear in the coupling which distinguishes our situation from a Fermi-Pasta-Ulam dynamics [18].

In a linear regime the chain solutions can be expressed in terms of plane waves (phonons); i.e. $q_{n}(t)=$ $q_{0} \exp [i(k n-\omega t)]+$ c.c. obeying the dispersion relation $\omega^{2}=\omega_{0}^{2}+4 \kappa \sin ^{2}(k / 2)$ with wave numbers $k=2 \pi k_{0} l / L$. Therein $l$ denotes the chain spacing, $L$ is the length of the chain yielding $L=l N$ and $k_{0}$ are the integers in the interval $k_{0} \in(-N / 2, N / 2]$.
The potential barrier in our set-up is given by

$$
\Delta E=\frac{\omega_{0}^{6}}{6 a^{2}} .
$$

A linear regime holds true in the considered potential (1) for sufficiently small energy of all chain members as compared to the potential barrier, yielding a quasiharmonic motion in phase space. A chain located initially in the metastable potential minimum will remain there for a very long time and jumps over the barrier occur at time scales of the Poincaré recurrence time. For a set-up with interacting strength $\kappa$ the barrier height can be estimated by assuming that only one unit of the chain is elongated which yields a value $\Delta E=\left(\omega_{0}^{2}-4 \kappa\right)\left(\omega_{0}^{2}+\right.$ $2 \kappa)^{2} /\left(6 a^{2}\right)$. Compared to the isolated unit a unit coupled to its neighbors experiences an increase of the barrier height $\Delta E$ of the order $4 \kappa^{2}$.

In clear contrast, in the nonlinear regime an initial state near the metastable minimum is structurally unstable as will be elucidated below. In order to enhance the nonlinear modes in the dynamics (3) with the potential (1) we propose the following scenario:

- An amount of energy $E_{0}=E_{\text {total }} / N$ is applied per unit which allows the activation of nonlinear, cooperative excitations of the chain.

By doing so, the units evolve nonlinearly and these nonlinear modes localize energy and do not interact with the low-energy phonon modes which prevents that the total energy becomes homogeneously re-distributed [13].

Thus, the chain possesses a total energy $E_{\text {total }}=N E_{0}$. For an escape to take place we must require that $E_{\text {total }}>$ $E_{\text {crit }}>\Delta E$. These inequalities convey the fact that more than just one unit governs the escape mechanism. The initial energy $E_{0}$ is supplied as follows: i) First, the whole chain is elongated homogeneously along a fixed position $q_{0}$ near well bottom. ii) Then, the position of all units and their momenta are iso-energetically randomized while keeping the total energy a constant; i.e. $E_{\text {total }}=$ $N E_{0}=$ const. The random position values are chosen from a bounded interval $\left|q_{n}(0)-q_{0}\right| \leqslant \Delta q$ and, likewise the random, initial momenta, $\left|p_{n}(0)\right| \leqslant \Delta p$. The whole chain is thus initialized close to an almost homogeneous state but yet sufficiently displaced $(\Delta q \neq 0)$ in order to generate non-vanishing interactions, enabling the exchange of energy among the coupled units.

The initial energy per unit obeys $E_{0} \ll \Delta E$, but is still sufficiently large to initiate the excitation of nonlinear modes. Then, the nonlinear part of the potential causes a modulational instability; perturbations with a wave number $Q$ may grow exponentially, resulting in a subsequent accumulation of energy at the expense of the energy from the other units. For our described initial set-up this growth proceeds with the rate [19]

$$
\Gamma=\sin \left(\frac{Q}{2}\right)(8 \kappa)^{1 / 4} \sqrt{\left(\frac{10 a^{2}}{3 \omega_{0}^{2}} q_{0}^{2}-\frac{\kappa}{\sqrt{2}} \sin ^{2}\left(\frac{Q}{2}\right)\right)} .
$$




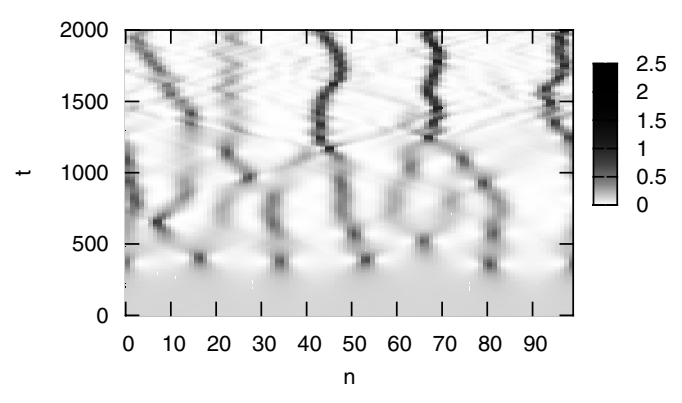

Fig. 1: Density plot of the energy of individual units $n$, illustrating the formation of a localized structure upon evolving time $t$. The color scale indicates the energy content, with dark grey corresponding to a high-energy regime. The chain consists of 100 units and the initial set-up is realized by setting: $q_{0}=0.35, \Delta q=0.01, p_{0}=\Delta p=0$. The remaining parameters are $\omega_{0}^{2}=2, a=1$, and $\kappa=0.3$. The total energy amounts to $E_{\text {total }}=8.1 \times \Delta E$. Note the strong energy localization due to breather coalescence around the units with number $n=45$ and $n=69$.

Because the argument of the square root must assume positive values the equipartition is unstable whenever

$$
\frac{a^{2} q_{0}^{2}}{\kappa \omega_{0}^{2}}-\frac{3}{10 \sqrt{2}} \sin ^{2}\left(\frac{Q}{2}\right)>0 .
$$

To investigate the formation of intrinsically localized modes in our discrete system the set of coupled equations (3) has been numerically integrated by use of a fourth-order Runge-Kutta scheme. The accuracy of the calculation is tested by monitoring the conservation of the total energy at a precision level of $10^{-5}$. In our simulations the chain consists of $N=100$ coupled nonlinear oscillators.

Starting out from an initial, nearly homogeneous state with an approximate equipartition of energy among all units the attainment of an array of breathers is observed. More precisely, due to the emergence of a modulational instability a pattern evolves in the course of time (of the order of $t \sim 2 \times 10^{3}$ ) where for some lattice sites the amplitudes grow considerably remaining small in the adjacent regions. This feature is depicted with fig. 1 . The breather states possessing a relatively high energy occur spontaneously at an average distance of the inverse wave numbers $Q_{\max }^{-1}$, corresponding to the maximal growth rate $\Gamma_{\max }$ in (5). Upon moving, these breathers tend to collide inelastically with others. In fact, various breathers merge to form larger amplitude breathers, proceeding preferably such that the larger amplitude breathers grow at the expense of the smaller ones. As a result, a certain amount of the total energy becomes strongly concentrated within confined regions of the chain. This localization scenario, as demonstrated here for the case of a metastable cubic potential, is characteristic for other nonlinear lattice systems as well $[15,19,20]$. By construction the initial total energy $E_{\text {total }}=10.821$ is almost uniformly shared among all units. In fig. 1 the ratio of initial energy $E_{0}$ and the
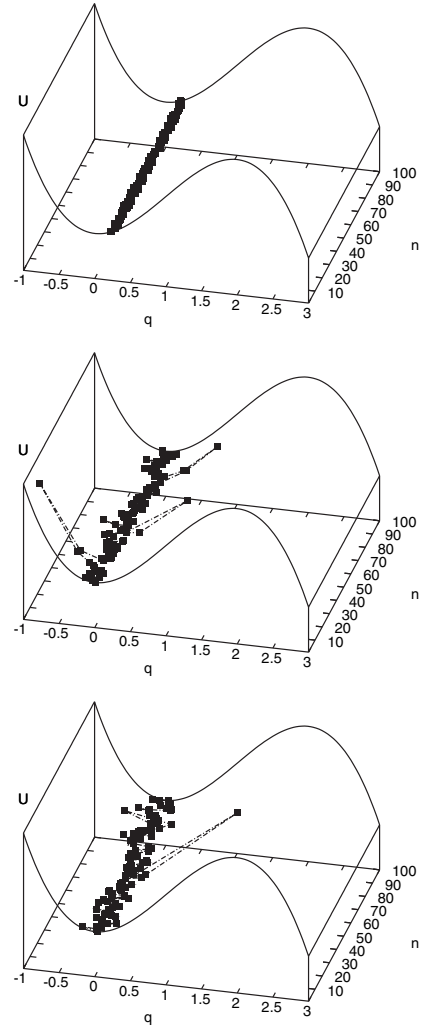

Fig. 2: Typical behavior of the chain placed in the cubic potential. Snapshots of the potential energy $U\left(\left\{q_{n}(t)\right\}\right)$ illustrate the formation of several breathers, which subsequently merge to concentrate the energy so that at least one unit can approach the barrier. Parameters are as in fig. 1 but here we have $\Delta q=0.1$. Top panel: $t=0$, initial state of nearly homogeneous energy distribution; central panel: $t=913$, emergence of localized structures; and, bottom panel: $t=1922$, strongly localized state with one unit near the barrier.

barrier energy is $E_{0} / \Delta E=0.081$. Thus, a single unit must acquire the energy content of more than 12 other units before it can overcome the barrier.

A breather solution may grow sufficiently strong in amplitude so that the energy barrier can be surmounted. To relate the energy localization with escape over the barrier we depict in fig. 2 snapshots of $U\left(\left\{q_{n}(t)\right\}\right)$ at different instants of time. In the beginning the energy is virtually equally shared among all units (not shown). After a certain time, the local energy accumulation is such enhanced that at least one of the involved units possess enough energy to overcome the barrier. The question then is: does an escaped unit continue its flight beyond the barrier or can it even be pulled back into the bound chain formation $\left(q_{n}<q_{\max }\right)$ by the restoring binding forces exercised by its neighbors? On the other hand, the unit that has already escaped from the potential well might drag neighboring ones closer to or in the extreme even over the barrier. Thus, concerted escape of at least parts of the chain, if not the whole chain, from the potential valley seems possible. 


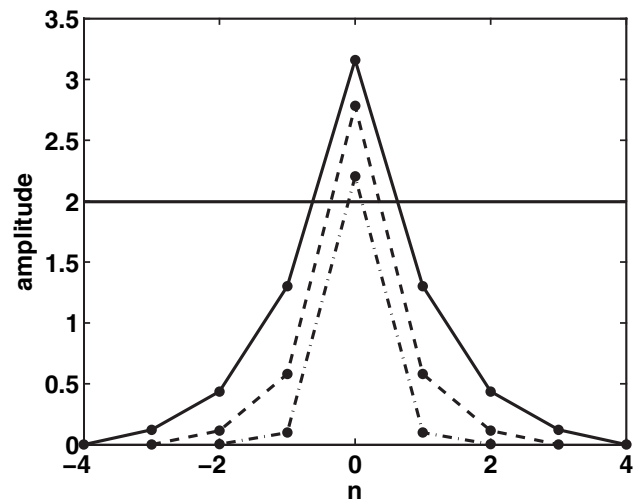

Fig. 3: The amplitude profile is depicted for a critical chain configuration, being symmetrically centered at $n=0$, for different coupling strengths: $\kappa=0.1$ (dash-dotted line), $\kappa=0.5$ (dashed line), and $\kappa=1$ (solid line). Note that for better illustration only a small part of the lattice chain with seizable elongations is shown. The chosen parameters are $\omega_{0}^{2}=2$, $a=1$ for a chain with $N=100$.

Whether a single unit of growing amplitude can escape from the potential well or merely is dwelling the well region via the action of the restoring forces of its neighbors depends both on the corresponding amplitude ratio and on the coupling strength.

An individual critical chain configuration $\tilde{q}_{n}$, reaching the transition state that separates bounded from unbounded solutions is determined by solving $\ddot{\tilde{q}}_{n}(t)=0$ and $\dot{\tilde{q}}_{n}(t)=0$. The relation in $(3)$ reduces to $-\partial U / \partial \tilde{q}_{n}+$ $\kappa\left[\tilde{q}_{n+1}+\tilde{q}_{n-1}-2 \tilde{q}_{n}\right]=0$. This equation can be derived from the potential energy function $E_{\text {pot }}\left(\left\{\tilde{q}_{n}\right\}\right)=$ $\sum_{n}\left(U\left(\tilde{q}_{n}\right)+\frac{\kappa}{2}\left[\tilde{q}_{n}-\tilde{q}_{n-1}\right]^{2}\right)$, yielding in the critical configuration a vanishing force, i.e. $\partial E_{\text {pot }} / \partial \tilde{q}_{n}=0$. The corresponding solution of this minimal critical energy assumes on the lattice chain the form of a localized hump which resembles the form of a hairpin.

In fig. 3 profiles of this hairpin-like, stationary critical localized mode (c.l.m.), or critical nucleus, with positions located at $\left\{\bar{q}_{n}\right\}$ and total energy $E_{c r i t}$ are depicted for several coupling strengths. Note also that a kink-like c.l.m.-configuration, being possible with open-end boundary conditions [21], is ruled out here in our case with periodic boundary conditions. We observe that the stronger is the coupling the larger is the maximal amplitude of the hump and the wider is the spatial extension of the latter. We underline that on a sufficiently extended lattice this (c.l.m.)-mode represents a narrow chain formation with its width being much smaller than the total chain length. Apparently, rising the coupling strength $\kappa$ causes an increase of the critical energy, $E_{c r i t}$. Because this unstable critical equilibrium solution $\left\{\tilde{q}_{n}\right\}$ constitutes a "forcefree" chain configuration we find that upon evolving time an overall positive force acts on all units which in turn yield a unidirectional escape into the neighboring attracting well region.

Therefore, if the kinetic energy overcomes the critical nucleus the subsequent escape of the neighbors is initiated,

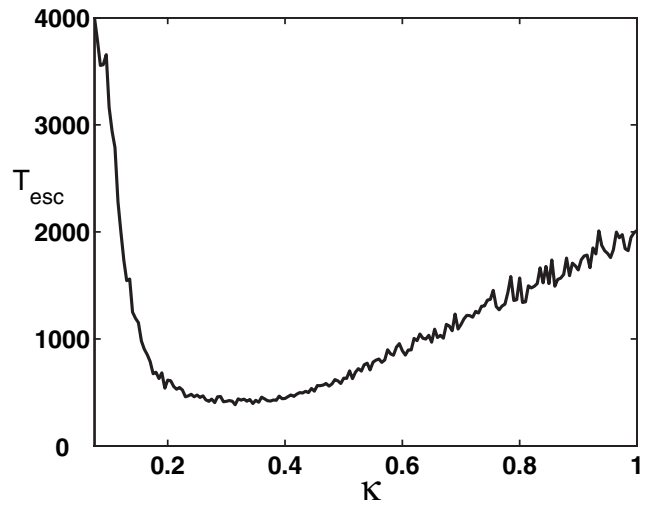

Fig. 4: Noise-free, mean escape time $T_{e s c}$ as a function of the coupling strength $\kappa$. The remaining parameters are as in fig. 1 except for $q_{0}=0.45, \Delta q=0.1$. The averages are performed over 1000 realizations of the initial conditions.

which progress on the chain to the left and to the right of the hair pin as a propagating kink and anti-kink, respectively, see refs. $[6,22,23]$. In this manner an efficient lowering of the total potential energy is accomplished. Since the kinetic energy of this outward motion consequently increases a return backwards over the barrier into the original well is prevented.

The escape time $T_{e s c}$ for the chain is defined as the average of the moments at which the $N$ amplitudes of the escaping units pass a certain value $q$ beyond the barrier location. Here, we chose $q=10$, i.e. $q=2.5 q_{\max } . T_{\text {esc }}$ $v s$. the coupling strength is displayed in fig. 4. An efficient escape is crucially influenced by the fast growing perturbational mode with the wave number $Q_{\max }=2 \pi N_{\max } l / L$ determining the number of units, $N_{\max }$, which belong to the emerging localized pattern, cf. eq. (5). The optimal strategy for fast escape is then that one of the emerging humps accumulates an energy larger than $E_{\text {crit }}$. In this case the localized pattern is provided already by the mechanism of modulational instability. Otherwise, if $N_{\max } E_{0}<E_{\text {crit }}$ further energy accumulation due to breather coalescence is needed which only will inhibit a speedy escape process. The relation (5) reveals that for the optimal coupling strength $\kappa \simeq 0.31$ the growth rate assumes a maximum $\Gamma_{\max }$ at a wave number $Q_{\max } \simeq 1.25$. This in turn induces the minimal escape time depicted in fig. 4.

Let us also compare the noise-free escape process with a corresponding thermally activated process $[1,6-8,22,23]$. The corresponding Langevin dynamics reads

$$
\frac{\mathrm{d}^{2} q_{n}}{\mathrm{~d} t^{2}}+\gamma \frac{\mathrm{d} q_{n}}{\mathrm{~d} t}+\frac{\mathrm{d} U}{\mathrm{~d} q_{n}}-\kappa\left[q_{n+1}+q_{n-1}-2 q_{n}\right]+\xi_{n}(t)=0
$$

Here $\gamma$ is the friction parameter and $\xi_{n}(t)$ denotes a Gaussian distributed thermal random force of vanishing mean and correlation $\left\langle\xi_{n}(t) \xi_{n^{\prime}}\left(t^{\prime}\right)\right\rangle=2 \gamma k_{B} T \delta_{n, n^{\prime}} \delta\left(t-t^{\prime}\right)$. Our numerical simulations results for the escape of the chain are depicted with fig. 5. We took averages of 


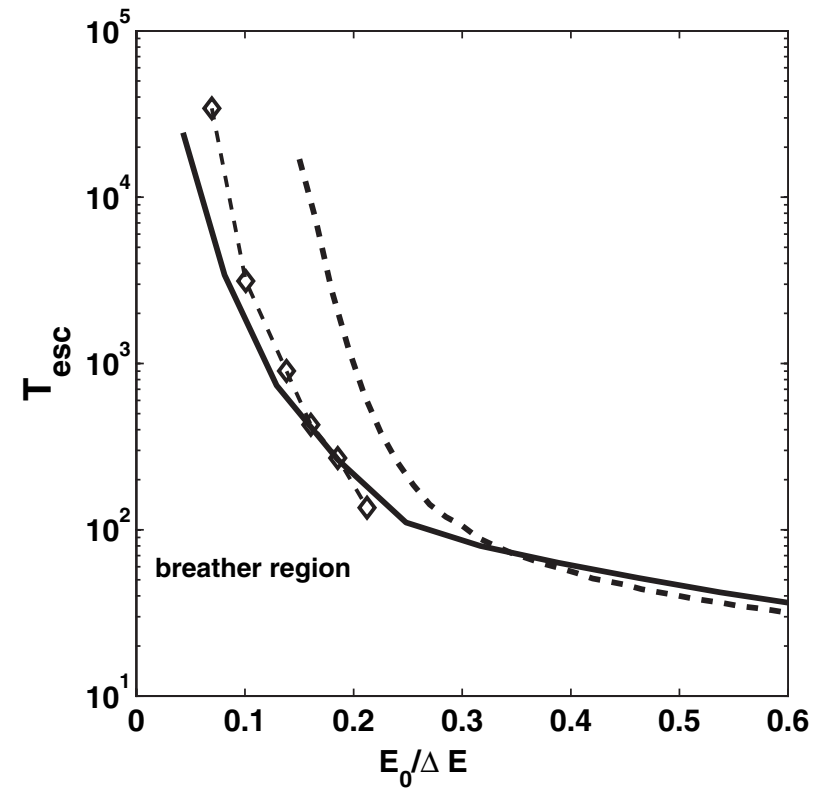

Fig. 5: Mean escape time $T_{\text {esc }}$ vs. the ratio of energy per unit and barrier energy, i.e. $E_{0} / \Delta E$ for the noise-free case $k=0$ mode (solid line), randomized initial conditions (diamonds) and noise-assisted case (dashed line), respectively. The parameters are: $q_{0}=0.40, \Delta q=0.1, \omega_{0}^{2}=2, a=1, \kappa=0.3$ and $\gamma=0.1, N=100$.

1000 realizations of random initial conditions in the microcanonical case and for the noisy escape trajectories of the Langevin equations, respectively. The Langevin equations were numerically integrated using a two-order Heun stochastic solver [24] as a function of the ratio $E_{0} / \Delta E$. For the noise-free, deterministic system (3) $E_{0}$ is given by the initial energy per unit which is chosen to correspond to thermal energy $k_{B} T$ in case of the Langevin dynamics (7). In both cases there occurs an increasingly fast escape, i.e. $T_{\text {esc }}$ becomes increasingly shorter upon an increasing ratio $E_{0} / \Delta E$. Most remarkably, for low ratios $E_{0} / \Delta E$ (the region indicated as the "breather region" in the figure) the escape occurs distinctly faster for the noise-free case as for a situation of a chain that is coupled to a heat bath. This implies a large enhancement of the rate of escape as compared to the thermal rate. Near $E_{0} / \Delta E \geqslant 0.36$ there occurs a crossover, with the mean escape time of the deterministic system at even higher ratios closely following that of the Langevin dynamics.

To sharpen our finding that the escape proceeds typically faster in the noiseless situation as compared to the case with a heat bath, we investigated also the escape process of chain patterns starting out from non-flat and even purely randomized initial conditions. First, we briefly report on our simulations performed for non-flat initial structures, i.e. for which the initial state is represented by a plane wave $q_{n}=u_{0} \exp (i k n)+$ c.c. with wave number $k \neq 0$ yielding a pattern that complies with the periodic boundary conditions. The value of the amplitude $u_{0}$ was chosen such that for all considered $k$ values the system assumes the same total energy. To summarize the results concerning the mean escape time, we found that for larger wave numbers $k$ the escape process becomes prolonged. For $k=\pi / 10$, as the largest wave number for which escape was observed within our simulation time-span of $10^{5}$, we found that $T_{e s c}=7839$, which lies still markedly below the escape time in the thermally activated situation corresponding to an equal ratio $E_{0} / \Delta E=0.1$. Note also that there is no modulational instability for short wavelength patterns belonging to wave numbers $k \in[\pi / 2, \pi][19,21]$.

For randomized initial conditions, where the coordinates and momenta are distributed in a fairly broad range $\Delta q$ and $\Delta p$, the initial energy contents of the individual units are widely distributed around a mean value $E_{0}$. In comparison for the above-discussed case with the $k=0$ mode, the differences in the initial energy content of the individual units are small due to a narrow distribution range $\Delta q$. The findings for the mean escape time as a function of the mean initial energy content of the units relative to the barrier height $E_{0} / \Delta E$ are included in fig. 5 as diamond symbols. For each ratio $E_{0} / \Delta E$ the averages were taken over 100 realizations of the random distributions of the coordinates and momenta. Most importantly, even for random initial conditions the mean escape time assumes smaller values in the microcanonical situation as compared to the Langevin dynamics. This underpins our general statement that noiseless escape indeed proceeds faster than thermally activated escape.

We note that the breathers present robust chain configurations that are formed rather fast as compared to the escape time. In contrast, the forever impinging stochastic forces impede such a fast growth of the critical nucleus and cause even the possible destruction of the critical chain formation, causing re-crossings of the transition region which only hamper a speedy escape. This inhibition for escape is most effective at small ratios of $E_{0} / \Delta E$, being induced either by high barrier heights, or low temperatures (implying a small $E_{0}$ ). A deterministic scenario thus presents a more favorable route towards speedy escape in situations with very weak noise or very large barrier heights. Having performed also simulations for more general situations with i) non-harmonic, nonlinear chain interactions, ii) in higher dimensions and iii) differing on-site potentials we find [21] that the phenomenon of an enhanced, noise-free escape remains robust in regimes of small ratios between initial energy per unit $E_{0}$ and corresponding barrier height $\Delta E$.

We in addition also studied systems which are more complex than the case of a one-dimensional chain model with harmonically interacting units [21]. It turned out that i) for chain systems with more than one degree-offreedom per unit (e.g. taking into account also motions of the units along the direction of the chain axis) and ii) for interactions going beyond the linear (harmonic) one (such as, e.g., with Morse-type interaction potentials) the escape time in the microcanonical situation typically is 


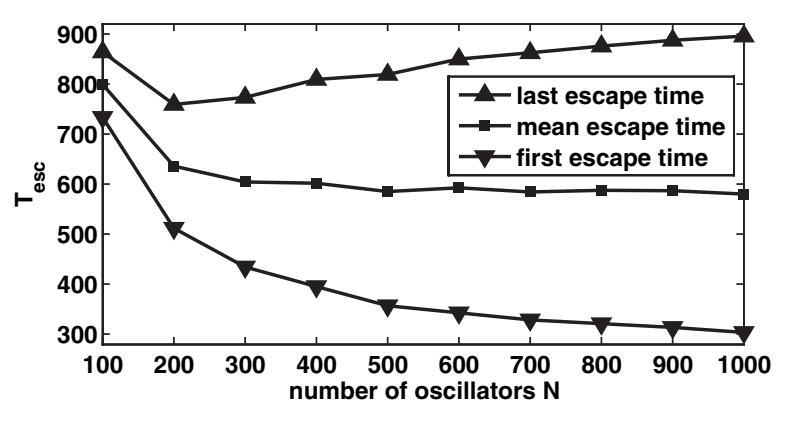

Fig. 6: First, mean and last escape time $T_{e s c}$ as a function of the chain length $N$. The parameters are: $q_{0}=0.50, \Delta q=0.01$, $\omega_{0}^{2}=2, a=1, \kappa=0.2$.

significantly shorter than for the situation with the system being coupled to a heat bath.

The interesting role of the chain length on the escape process has been studied with fig. 6: Therein, the first, the average and the last escape time are depicted $v s$. varying chain length with constant energy density $\rho=E_{\text {total }} / N=$ const. Apparently the longer the chain, the more humps (breathers) are formed due to the modulational instability. This in turn offers the possibility that an enlarged number of interacting breathers contribute to an enhanced energy localization in a confined region of the chain that enhance the formation of the critical localized mode. Thus, the time it takes for the first unit to escape shrinks with increasing chain length; but eventually saturates with increasing chain length, see in fig. 6. Because the kink-antikink propagates with a constant velocity, the mean escape time becomes insensitive to variations of the chain length for sufficiently large lengths $N \geqslant 500$.

In summary, we studied noise-free escape in a chain of coupled oscillators. While at weak thermal noise the rate of thermal escape is exponentially suppressed, a deterministic nonlinear breather dynamics yields a robust critical nucleus configuration, which in turn causes an enhancement of the noise-free escape rate. Thus, the freezingout of noise may prove advantageous for transport in metastable landscapes, whenever the deterministic escape dynamics can be launched in a single shot via an initial energy supply.

\section{$* * *$}

This research was supported by SFB-555 (LSch-G, $\mathrm{DH})$; the Volkswagen Foundation Project I/80424 (PH) and I/80425 (LSch-G). The authors are grateful to S. Fugmann for the preparation of fig. 1 and fruitful discussions.

\section{REFERENCES}

[1] Hänggi P., Talkner P. and Borkovec M., Rev. Mod. Phys., 62 (1990) 251.

[2] Langer J. S., Ann. Phys. (N.Y.), 54 (1969) 258.
[3] Sung W. and Park P. J., Phys. Rev. Lett., 77 (1996) 783.

[4] Park P. J. and Sung W., Phys. Rev. E, 57 (1998) 730; J. Chem. Phys., 108 (1998) 3013; 111 (1999) 5259.

[5] Dikshtein I. E., Polzikova N. I., Kuznetsov D. V. and Schimansky-Geier L., J. Appl. Phys., 90 (2001) 5425; Dikshtein I. E., Kuznetsov D. V. and Schimansky-Geier L., Phys. Rev. E, 65 (2002) 061101.

[6] Sebastian K. L. and Paul A. K. R., Phys. Rev. E, 62 (2000) 927.

[7] Lee S. and Sung W., Phys. Rev. E, 63 (2001) 021115; Lee K. and Sung W., Phys. Rev. E, 64 (2001) 041801.

[8] Kraikivsky P., Lipowsky R. and Kiefeld J., Europhys. Lett., 66 (2004) 763; Eur. Phys. J. E, 16 (2005) 319.

[9] Dowtown M. T., Zuckermann M. J., Craig E. M., Plischke M. and Linke H., Phys. Rev. E, 73 (2006) 011909.

[10] Hänggi P., Marchesoni F. and Nori F., Ann. Phys. (Berlin), 14 (2005) 51.

[11] Rabinovich M. I., Varona P., Selverston A. I. and Abarbanel H. D. I., Rev. Mod. Phys., 78 (2006) 1213.

[12] Sato M., Hubbard B. E. and Sievers A. J., Rev. Mod. Phys., 78 (2006) 137.

[13] Flach S. and Willis C. R., Phys. Rep., 295 (1998) 181.

[14] Takeno S. and Tsironis G. P., Phys. Lett. A, 343 (2005) 274.

[15] Kivshar Yu. S. and Peyrard M., Phys. Rev. A, 46 (1992) 3198; Peyrard M., Physica D, 119 (1998) 184; Dauxois T., Ruffo S. and Torcini A., Phys. Rev. E, 56 (1997) R6229; Cretegny T., Dauxois T., Ruffo S. and Torcini A., Physica D, 121 (1998) 109.

[16] Hennig D. and Tsironis G. P., Phys. Rep., 307 (1999) 333.

[17] Lazarides N., Eleftheriou M. and Tsironis G. P., Phys. Rev. Lett., 97 (2006) 157406; IvANChenko M. V., Kanakov O. I., Mishagin K. G. and Flach S., Phys. Rev. Lett., 97 (2006) 025505; Flach S., IVANChENKo M. V. and Kanakov O. I., Phys. Rev. E, 73 (2006) 036618; Cuevas J., Kevrekidis P. G., Frantzeskakis D. J. and Bishop A. R., Phys. Rev. B, 74 (2006) 064304; Flach S. and Gorbach A., Int. J. Bifurcat. Chaos, 16 (2006) 1645; Maniadis P. and Flach S., Europhys. Lett., 74 (2006) 452; Aubry S., Physica D, 216 (2006) 1.

[18] Berman G. P. and Izrailev F. M., Chaos, 15 (2005) 015104.

[19] Daumont I., Dauxois T. and Peyrard M., Nonlinearity, 10 (1997) 617.

[20] Eleftheriou M. and Tsironis G. P., Phys. Scr., 71 (2005) 318.

[21] Hennig D., Fugmann S., Schimansky-Geier L. and HÄNGGI P., in preparation.

[22] Petukhov P. V. and Pokrovskit V. L., Sov. Phys. JETP, 36 (1973) 336 (Zh. Eksp. Teor. Fiz., 63 (1972) 634); Büttiker M. and Landauer R., Phys. Rev. A, 23 (1981) 1397.

[23] Hänggi P., Marchesoni F. and Sodano P., Phys. Rev. Lett., 60 (1998) 2563; HÄNGGi P. and MARChESONI F., Phys. Rev. Lett, 77 (1996) 787.

[24] CARD T. C., Introduction to Stochastic Differential Equations, Chapt. 7, Monographs in Pure and Applied Mathematics, Vol. 114 (M. Dekker Inc., New York) 1988. 